\title{
La Chine en débat
}

\section{Thomas BOUTONNET}

\section{OpenEdition \\ Journals}

Édition électronique

URL : http://journals.openedition.org/transtexts/401

DOI : 10.4000/transtexts.401

ISSN : 2105-2549

\section{Éditeur}

Gregory B. Lee

Édition imprimée

Date de publication : 7 avril 2011

ISSN : 1771-2084

\section{Référence électronique}

Thomas BOUTONNET, "La Chine en débat », Transtext(e)s Transcultures 跨文本跨文化 [En ligne], 6 | 2011, mis en ligne le 05 avril 2011, consulté le 22 septembre 2020. URL : http://

journals.openedition.org/transtexts/401 ; DOI : https://doi.org/10.4000/transtexts.401

(C) Tous droits réservés 


\section{Transtext(e)s}

Transcultures 跨文本跨

文化

Journal of Global Cultural Studies

6 | 2011:

Debating China

\section{La Chine en débat}

\section{THOMAS BOUTONNET}

\section{Texte intégral}

Le spectacle n'est pas un ensemble d'images, mais un rapport social entre des personnes, médiatisé par des images. ${ }^{1}$

Guy Debord posait en 1967 dans la Société du spectacle la question, ouverte, de la mythification de la réalité, de la vie par procuration mais également, et surtout, de la misère d'une vie quotidienne réglée et régulée par l'unique satisfaction d'un ensemble de critères prédéfinis (la consommation de biens, le statut social), mis en images et expropriant l'individu de son temps et de la maitrise de son temps. Quarante ans plus tard, la question reste pertinente car l'emprise des images, des mises en scènes et du spectacle sur nos sociétés et sur nos existences est de plus en plus marquante. Or il n'est pas, personne n'en doute, d'images qui ne soient pas assujetties à un mode de pensée. La réalité se construit en termes d'images et de représentations, de récits et de mythes, autant d'éléments qui nous permettent d'appréhender le réel mais qui nous en éloignent également, autant d'éléments qui contribuent nécessairement, directement ou non, à établir et à enraciner des positionnements idéologiques.

2 La genèse et la création de ces représentations et de ces images est probablement l'un des terrains de recherche les plus stimulants des sciences humaines et sociales depuis un demi siècle ; l'un des plus utiles également dans la compréhension des éléments qui constituent la post-modernité, ses moyens et ses limites. Et s'il est un pays au monde où la question de l'image se pose avec une pertinence et une gravité certaines, ce pays est sans nul doute, pour différentes raisons historiques, la Chine. Fantasme de l'imaginaire occidental 
depuis l'envoi des premières missions Jésuites, substrat de l'orientalisme défini par Said (et que la Chine commence à intégrer dans sa construction identitaire officielle), mais aussi « terrain d'exercice » des idéologies majeures du vingtième siècle où l'image joue un rôle essentiel (du Maoïsme au libéralisme économique), la Chine a vu dans son histoire se superposer et se sédimenter différentes couches de représentations qu'elle tente tant bien que mal d'unifier depuis une quinzaine d'années.

Objet protéiforme, fantasmé ou simplement imaginé, la Chine s'est finalement le plus souvent retrouvée éloignée dans sa propre représentation, réifiée et codifiée. Et si depuis l'émergence de la République populaire de Chine sur la scène diplomatique et économique mondiale au milieu des années 1990, l'image du pays - celle qu'il construit et renvoie de lui-même, combinée à celle que le monde, essentiellement les pays occidentaux, construit sur lui - se dirige lentement mais sûrement vers une forme de normalisation (la Chine est maintenant devenu un régime respectable, et on ne parle guère plus de totalitarisme), la question essentielle qui doit être posée reste : qu'est-ce que la Chine?

4 Debating China constitue donc le thème central et la première partie de ce sixième numéro de Transtext(e)s Transcultures, une partie dans laquelle cette question, " qu'est-ce que la Chine ? ", sera déclinée par le biais de quatre approches originales au croisement de différentes disciplines, quatre travaux sur les notions d'images, d'imaginaire et de représentation.

$5 \quad$ Pierre-Mong Lim se propose d'aborder cette question par le prisme de la notion de sinité. Étudiant deux productions hollywoodiennes récentes, Mulan et Kungfu Panda, l'auteur déconstruit le discours orientaliste à l'œuvre dans ces animations, questionne les mécanismes idéologiques et économiques qui président la constitution de cette identité chinoise mise en images, mais aussi, et surtout, interroge la dynamique de l'auto-orientalisme, c'est à dire de l'orientalisme intégré et reproduit par l'objet lui-même (la Chine).

$6 \quad$ La question de la Chine, de ses représentations, des images et des sensations qui lui sont associées se retrouve également déclinée dans l'article de Marilyne Brun qui étudie After China, un roman majeur dans l'œuvre de Brian Castro. La Chine s'y retrouve imagée en termes d'espaces et de temps (il y a un " après » la Chine) et dissociée en deux narrations opposées (la Chine ancienne, la Chine contemporaine) : deux Chines imaginées (et imaginaires ?) qui servent un propos critique sur la tragédie humaine que fut la Révolution Culturelle, mais qui articulent également une critique de la biopolitique et du contrôle des corps qu'elle implique.

$7 \quad$ Jean-Yves Heurtebise aborde lui les images et les discours sur la Chine sous l'angle philosophique et politique de la démocratie. Dépassant la réduction simpliste d'un Occident supposé berceau de la démocratie et d'un Orient prétendument conditionné par des régimes totalitaires ou autoritaires (rappelons que le régime chinois se qualifie lui-même de démocratique, et que la question du dévoiement de la démocratie est régulièrement soulevée dans les pays occidentaux), l'auteur questionne la définition même de cette construction politique tout en retraçant l'historique des discours européens sur la notion de démocratie et sur la possibilité d'une démocratie en Chine (et en Asie d'une manière générale). L'article s'interroge par ailleurs sur l'existence de "valeurs occidentales » et de «valeurs asiatiques » ainsi que sur la genèse de ces discours. 
Cette question de la démocratie (et de sa mise en représentations, en images et en discours) est également abordée par Huang Yu dans le dernier article de cette partie Debating China. Il s'agit cette fois d'un débat non pas sur la Chine mais en Chine, puisque Huang Yu analyse une controverse publique virulente qui eut lieu en 2005 autour de l'émission Chaoji Nusheng 超级女声 (Super Girl Voice Contest). Cette émission télévisée très populaire (un concours de chant inspiré d'une émission américaine) fut à l'époque le prétexte à un débat engagé entre intellectuels chinois sur la question de l'exercice et de la pratique de la démocratie, sur la relation entre culture populaire et société de consommation, mais également, et plus généralement, sur le rôle des intellectuels dans une Chine contemporaine coincée entre libéralisme économique et conservatisme politique.

$9 \quad \mathrm{Xu}$ Kefei aborde également la question centrale du rôle des intellectuels dans la narration de l'histoire et la transformation du présent. Il nous explique que l'engouement des rédacteurs de la revue Tel Quel (1960-1982) pour la pensée maoïste en général et la Révolution culturelle en particulier n'est qu'une nouvelle déclinaison d'une approche, très française, de la Chine comme miroir.

La deuxième partie de ce numéro de Transtext(e)s Transcultures répond à l'une des exigences fondamentales de la revue : proposer un espace d'expression aux travaux scientifiques transdisciplinaires débarrassés des carcans catégoriels et capables de s'aventurer sur des terrains de recherche dans une approche transculturelle. Ainsi, cette deuxième partie inclut trois contributions originales regroupées dans un Varia, trois approches « transnationales » qui toutes explorent, au travers de penseurs majeurs du siècle dernier, la question fondamentale de l'identité.

Dans un premier article, Liu Huiming étudie le travail de Paul Ricoeur sur les questions du soi et de l'identité, et explore sa contribution majeure en la matière : la notion d'identité narrative, articulation dialectique de l'identitéipséité et de l'identité-mêmeté.

12 Dans un second article, Le Min Sook étudie l'œuvre de l'écrivain coréen Kim Dong-ri, et plus particulièrement la représentation du chamanisme dans ses écrits. Le chamanisme coréen s'y affirme comme une réponse à la modernité et aux bouleversements qu'elle implique, et l'article interroge ce corpus d'idées qui, par delà ses aspects conservateurs, se présente comme un troisième humanisme (après celui de la Grèce ancienne et celui de la Renaissance).

13 Enfin, le dernier article de ce Varia, signé de Ma Jin, aborde l'œuvre de Jean Baudrillard, et son approche des notions de signes et de sens. Jean Baudrillard, le plus souvent étudié pour son travail la société de consommation, a également contribué à une approche critique de la sémiologie que l'article se propose d'exposer, tout en interrogeant les concepts de simulacre et de simulation.

Dans notre époque de plus en plus inhibée par les enjeux économiques et sclérosée par une logique productiviste, où « le monde sensible se trouve remplacé par une sélection d'images qui existe au-dessus de lui », il est plus que jamais nécessaire de déconstruire les modèles de pensée et de représentations de manière à sortir les imaginaires des carcans idéologiques et politiques dans lesquels ils sont généralement contraints. ${ }^{2}$ Transtext(e)s Transcultures se veut être une opportunité de décloisonner les pensées, étape indispensable à une reformulation émancipée du monde et de ses représentations. 


\title{
Notes
}

1 Guy Debord, La Société du spectacle, Paris, Gallimard, 1992, p.4.

2 Guy Debord, La Société du spectacle, Paris, Gallimard, 1992, p.21.

\section{Pour citer cet article}

Référence électronique

Thomas BOUTONNET, «La Chine en débat », Transtext(e)s Transcultures 跨文本跨文 化 [En ligne], 6 | 2011, mis en ligne le 05 avril 2011, consulté le 08 mars 2012. URL : http://transtexts.revues.org/401

\begin{abstract}
Auteur
Thomas BOUTONNET

Thomas BOUTONNET est docteur en études chinoises et transculturelles, et a été directeur de l'Institut Confucius de Lyon (2009-2010). Chercheur au sein de l'Institut d'Études Transtextuelles et Transculturelles (www.iett.eu), il enseigne actuellement la langue et la société contemporaine chinoises à l'université de Lyon - Jean Moulin. Sa thèse, intitulée "Vers une "société harmonieuse" de consommation ? Discours et spectacle de l'harmonie sociale dans la construction d'une Chine "civilisée" (1978-2008) ", aborde le processus de civilisation des populations paupérisées durant le passage à une économie de marché en Chine. Son travail de recherche actuel porte sur la déconstruction des discours idéologiques en Chine contemporaine, sur l'étude des mots du pouvoir chinois et sur une analyse approfondie du concept de civilisation. Thomas BOUTONNET has a PhD in Chinese and Transcultural Studies and was the executive director of the Lyon Confucius Institute in 2009-2010. He currently lectures in the Chinese department of the University of Lyon - Jean Moulin and is an active member of the Institute for Transtextual and Transcultural Studies (www.iett.eu). His main research interests include ideology and discourse in contemporary China, Chinese official and political language, technologies of power, and the discourses surrounding the idea of "civilisation". His PhD thesis, entitled "Towards a 'Harmonious' Consumer Society? The Discourse and Spectacle of Social Harmony in the Construction of a 'Civilized' China (1978-2008)", focused on Hu Jintao's concept of a "harmonious society" and its relation to the development of consumerism.

Articles du même auteur

Debating China [Texte intégral]

Editorial [English version]

Paru dans Transtext(e)s Transcultures 跨文本跨文化, 6 | 2011

Traitement moral de la question sociale dans la « société harmonieuse » de Hu Jintao [Texte intégral]

Paru dans Transtext(e)s Transcultures 跨文本跨文化, 5 | 2009
\end{abstract}

\section{Droits d'auteur}

(C) Tous droits réservés 